\title{
The effects of natural category size on memory for episodic encodings
}

\author{
DOUGLAS L. NELSON, JOSE CANAS, and MARIA-TERESA BAJO \\ University of South Florida, Tampa, Florida
}

\begin{abstract}
Previous findings indicate that natural category size affects cued recall but not recognition performance. Words that define or belong to larger categories are not as likely to be recalled in the presence of an extralist cue. However, category size has no effect on recognition in the presence of the target as the cue. Theoretically, this difference could be due to inherent differences between these tasks, to the use of different types of test cues, or to differences in the nature of the required responses (naming compared with "yes/no"decisions). Three experiments indicated that none of these factors is a sole determinant. Natural category size effects were found in cued-recall and recognition tasks, with extralist and target cues and regardless of the required response. The critical factor is whether the testing conditions require or encourage subjects to search the category defined by the cue. With the initiation of such a search, information represented in semantic memory is likely to influence memory for episodic information.
\end{abstract}

Each word of a study list in a memory experiment is likely to become associated with the general experimental context and with other words in the list (e.g., Anderson \& Bower, 1974; Smith, Glenberg, \& Bjork, 1978). Both general contextual associations and interitem connections are episodic in the sense that, from the subjects' perspective, they are unique in time and place (Tulving, 1983). This episode, however, is not isolated from past experience. Each presentation of a familiar word or target activates or primes related concepts represented in long-term or semantic memory (e.g., Collins \& Loftus, 1975; Meyer \& Schvaneveldt, 1971; Underwood, 1965). To the extent that these related concepts become linked to the episodic experience or are otherwise activated in the task, they can assume an important role (Nelson, 1981). For example, target words that are known to be preconnected to many related concepts are not as likely to be recalled as are those that are preconnected to only a few related concepts (e.g., Nelson \& Bajo, 1985; Nelson, Bajo, \& Casanueva, 1985). That is, concepts that are not presented by the experimenter but are implicitly activated by the target interfere with memory for the target, and the magnitude of this interference is correlated with the number of concepts in the related set.

This target set size effect can be explained by retrieval inhibition that emanates from events that occur during the study episode and that place greater or lesser burdens on the test cue during retrieval. The presentation of a familiar word leads to the activation and encoding of its related concepts, and the cued-recall task requires the production of the presented word that must be selected from this pool of encoded concepts. The problem for the subject is to

This research was supported by Grant MH 16360 to the first author from the National Institute of Mental Health.

Requests for reprints should be sent to Douglas L. Nelson, Department of Psychology. University of South Florida, Tampa, FL 33620. produce the word actually studied rather than a word associated with an implicitly encoded concept. Presumably, studied words having larger sets of related concepts are not as likely to be recalled because there are more chances of retrieving nontarget items from the encoded set (e.g., Nelson, McEvoy, \& Friedrich, 1982; Raaijmakers \& Shiffrin, 1981; Roediger, 1974). In other terms, the retrieval inhibition explanation attributes the target set size effect to inhibitory processes associated with the retrieval of nontarget items during search.

This explanation suggests that retention tests that do not require search through the related concept set should not show the target set size effect. For example, in recognition testing, the retrieval cue consists of the studied word itself, which serves primarily as a prompt for its earlier occurrence in the study list (e.g., Watkins, 1979). With a lower probability, a target test word also may serve as a cue for the occurrence of other words in the study list belonging to the same category, and, in this case, small amounts of retrieval inhibition can be observed in recognition (Todres \& Watkins, 1981). Small deficits are therefore found in recognition when related concepts have been explicitly encoded as members of the study list. However, with target set size effects, category-related concepts are implicitly activated and are part of the study episode only by virtue of their being incidentally encoded. The target should not be as likely to be used as a cue to guide search through related concepts for evidence of occurrence. Therefore, target set size effects should not be readily apparent in recognition testing, and this appears to be the case (Nelson, Canas, Casanueva, \& Castano, 1985). This null effect is unlikely to be due to insensitivity, since over 16 experiments involving many variations in recognition testing conditions have been completed.

The contrast between cued recall and recognition indicates that natural category size effects will be found when memory for the target is prompted by an extralist cue but 
not when it is prompted by the target itself. The implication is that the difference between these two types of cues is what is critical. However, as Tulving (1983, chap. 14) noted, these tasks rely on different types of cues and on different types of responses. Cued recall involves nontarget cues with a request to name a specific target word from the studied list. Recognition involves the presentation of the target as a cue with a request to indicate whether it was present in the study list, usually with a "yes/no" response. In these task comparisons, type of cue and type of response are confounded, and conclusive statements about the influence of actual category size cannot be made.

The purpose of the present experiments was to compare directly the effects of type of cue and type of response to determine which factor is most closely linked to finding natural category size effects. The research design used in these experiments was a variation of one suggested by Tulving (1983, pp. 300-315). In Experiment 1, subjects were presented with a list of words, half of which were taken from large and half from small taxonomic categories. Subjects were told to remember the items and to think of a common category name for each item as it was presented (e.g., WINTER, think “Seasons"). During testing, type of test cue was manipulated by providing subjects with the target itself (e.g., WINTER) or by providing them with an extralist cue from the same taxonomic category (e.g., SUMMER). Type of response was manipulated by having subjects respond by indicating a "yes/no" decision or by naming the specific target. With this design, subjects giving a "yes/no" response in the presence of the target are being asked to perform a typical recognition task. Subjects who are naming the target when it is physically present are being asked to recognize the target by naming it. Those who are asked to name the target in the presence of an extralist cue are being asked to perform a typical cued-recall task. Finally, those asked to provide a "yes/no" decision in the presence of an extralist cue were asked for category recognition. For example, if WINTER was studied and SUMMER was shown at test, subjects were told to respond "yes" if they remembered that the category "Seasons" had been represented in the study list.

The primary question was whether category size effects would interact with type of response or with type of cue. An interaction with type of response would indicate that different responses may require different kinds of information for effective performance (Tulving, 1983). An interaction with type of cue showing category size effects for extralist but not for targets as cues would indicate that these effects are contingent on type of cue. In other words, regardless of the type of response, category size effects may be apparent whenever extralist cues are used. Extralist cues require subjects to search through related concepts in order to recover information encoded about the target (e.g., Raaijmakers \& Shiffrin, 1981). However, the critical factor in determining whether category size effects are found may be search per se. Even a target cue may be associated with category size effects when it is treated as an extralist cue that initiates the search process. Experiment 2 evaluated this possibility by replicating Experiment 1 and manipulating type of cue within, rather than between, subjects. Forcing subjects to switch randomly between extralist and target cues could cause them to treat at least some of the targets as extralist cues. In this case, category size effects should be apparent for both types of cues, and the previous contrast between cued recall and recognition would be attributed to differences in search demands associated with differences in the cues used in these two tasks. In general, extralist cues may be more likely to encourage search through related concepts. Finally, Experiment 3 represented an attempt to replicate the category size effect found with extralist cues in a procedure that involved recognition discrimination.

\section{EXPERIMENT 1}

\section{Method}

Design. The design formed a $2^{3}$ mixed-model factorial with type of response and type of cue manipulated between subjects and category size manipulated within subjects.

Materials. Twenty-four categories were taken from the McEvoy and Nelson (1982) norms. These categories and their associated instances are shown in Table 1. These norms were constructed by presenting subjects with a category name and asking them to produce the first word that came to mind that was a member of the category. This method, instead of the multiple-response procedure, was chosen because it avoids response chaining and retrieval inhibition effects and because it appears to provide a better measure of category size (Joelson \& Herrmann, 1978). Furthermore, correlational studies indicate that the relative strength of category members is preserved across the single- and multiple-response methods (e.g., Cofer, 1958; McEvoy \& Nelson, 1982).

Table 1 Experimental List

\begin{tabular}{ll}
\hline \multicolumn{1}{c}{ Category Name } & \multicolumn{1}{c}{ Instances } \\
\hline American Coin & PENNY, DIME \\
Branch of Armed Services & MARINES, NAVY \\
Bird of Prey & EAGLE, VULTURE \\
Musical Brass Instrument & TUBA, TROMBONE \\
Daily Meal & SUPPER, BREAKFAST \\
Part of a Face & MOUTH, CHEEK \\
Hair Color & BRUNETTE, AUBURN \\
Precious Gem & EMERALD, RUBY \\
Grammatical Part of Speech & PRONOUN, ADJECTIVE \\
Type of Singing Voice & BARITONE, TENOR \\
Branch of U.S. Government & JUDICIAL, LEGISLATIVE \\
Type of Reference Book & ENCYCLOPEDIA, THESAURUS \\
Type of Cloth & WOOL, SILK \\
Breed of Dog & COLLIE, SHEPHERD \\
Type of Fastener & ZIPPER, BUTTON \\
Felony Crime & ROBBERY, ARSON \\
Type of Flower & TULIP, DAISY \\
Fruit & PEAR, GRAPE \\
Kind of Liquor & WHISKEY, VODKA \\
Medical Specialty & SURGEON, NEUROLOGIST \\
Type of Nut & ACORN, PECAN \\
Type of Science & PHYSICS, ASTRONOMY \\
Professional Sport & TENNIS, HOCKEY \\
Wild Animal & TIGER, BEAR \\
\hline
\end{tabular}

Note-The first 12 categories define smaller and the last 12 categories define larger sets. 
Twelve of the categories selected for the experiment generated relatively large numbers of instances (mean $=21.83 ; S D=5.02$ ), and 12 produced smaller numbers of instances (mean $=6.08 ; S D$ $=1.38$ ). Two instances were taken from each category to serve as targets for the study list and as extralist test cues. These instances were of medium associative strength: An average of .11 ( $S D=$ .90 ) of the sample provided them as responses to the category name. Thorndike and Lorge (1944) frequency was equated for items falling into large and small categories (mean $=22$ per million words, G-count; $S D=24$ ).

Each of the category instances was normed independently by other samples of subjects (McEvoy \& Nelson, 1982). This scaling enabled determination of the overlap in responses for the category names and the instances (targets) and of the relationships among the targets themselves. The average of the Pearson product-moment corre lations between the sets of instances produced by the category name and the sets produced by the targets was $r=.73(S D=.24)$. This correlation was essentially the same for each target at each level of set size. Hence, the overlap in distributions tended to be high and it was equated over the set size manipulation. The set size defined by each target instance was held constant and averaged 8.12 $(S D=2.82)$ for those from small categories and $10.88(S D=2.82)$ for those from larger categories. Finally, the average strength of relation between instances from the same category was $.12(S D=$ $.11)$ for those from small categories and $.08(S D=.13)$ for those from larger categories. Hence, while category size was varied, both target set size and target-to-target strength were essentially equated within each level of category size.

In the target or copy cue conditions, the 24 targets also served as test cues. In the extralist cue conditions, the studied targets and test cues were switched (i.e., subjects studied one instance from a category and were tested on the other instance). In both target and extralist cue conditions, the test items were intermixed randomly with 24 distractors taken from categories not represented in the study list. Because of insufficient numbers of normed small and large categories, the distractors were taken from medium-sized categories on the assumption that the false recognition rate would not vary with category size.

Twenty-four items were also taken from other categories to serve as primacy and recency buffers for the study list. Hence, from the subjects' perspective, both the study list and the test list contained 48 items.

Procedure. Subjects participated in individual sessions and received a single study-test trial. All items were typed in uppercase letters and were presented by a Kodak carousel slide projector at a 3-sec rate. The study instructions required subjects to read the words aloud when shown, to think of a common category for each item as it was presented (e.g., WINTER, think "Seasons"), and to remember as many items as possible. They were not told how they would be tested until after the study phase. At this point. subjects were told different things depending on their condition. Those given "yes/no" instructions with target cues (recognition) were told that some of the words were from the study list and some were new. They were asked to indicate whether or not each presented item was a member of the study list by saying "yes/no. "Subjects given "yes/no" instructions with extralist cues (category recognition) were told that they were going to see some words that were categorically related to words from the study list and some new words. They were asked to respond "yes/no" to indicate if there was a word from the study list that belonged to the same category.

Subjects given naming instructions with target cues were told that they would be seeing clue words that would help them remember the list words. They were told that the clues were of two types: words from the study list and new words. They were asked to name the word from the study list, if any, that the clue word reminded them of. Finally, those given naming instructions with extralist cues (cued recall) were given the same instructions, except that they were told that the clue words were categorically related to the study list words. All subjects had to read aloud the cues before responding, and all instructions contained an example to facilitate understanding. The ordering of the items at study and test was changed for each subject in each condition.

Subjects. Sixteen subjects served in each of the between-subjects conditions, with 8 assigned to each target list, for a total of 64 subjects in the experiment. They were selected from courses in introductory psychology under an incentive system, and they were assigned randomly to conditions within replication block of 8 subjects.

\section{Results}

The results are presented in Table 2. In general, these results show that fewer items were named (.66) than were correctly judged "yes" (.77), that extralist cues (.55) were less effective than target cues (.87), and that performance was lower when category size was large (.67) than when it was small $(.76)$. Type of response $[F(1,60)=15.55$, $M S e=.029]$, type of cue $[F(1,60)=116.44]$, and category size $[F(1,60)=26.36, M S e=.013]$ were all significant sources.

These effects, however, were qualified by the reliability of two interactions, type of cue $\times$ category size $[F(1,60)=18.96]$ and type of cue $\times$ type of response $[F(1,60)=11.72]$. The interaction with category size is shown in the bottom row of Table 2 . The pattern clearly shows that category size affected memory performance when extralist cues were provided but not when targets were provided. The probability of judging "yes" or naming specific targets when extralist cues were shown was substantially lower when category size was large than when it was small. In contrast, when target cues were presented, performance was just as good when category size was large as when it was small. Thus, cue type was a critical determinant of category size effects. Neither the interaction between type of response and category size $[F=1.86]$, nor the three-way interaction involving these factors $[F=1.22]$ was reliable. Hence, type of response did not appear to influence differentially category size effects in any obvious way. This was confirmed by a separate analysis of the extralist cues, which showed reliable effects for type of response $[F(1,30)=26.31, M S \mathrm{Se}$ $=.030]$ and for set size $[F(1,30)=26.21, M S e=.022]$, but not for their interaction $(F=1.78)$. Despite the higher level of performance for the "yes/no" response, the magnitudes of category size effects were comparable in the two response conditions.

The interaction between type of cue and type of response indicated that response differences were found

Table 2

Probahility Correct as a Function of Type of Test Cue, Type of Response, and Category Size

\begin{tabular}{lcccc} 
& \multicolumn{3}{c}{ Type of Test Cue } \\
\cline { 2 - 5 } Type of Response & \multicolumn{2}{c}{ Target } & \multicolumn{2}{c}{ Extralist Cue } \\
\cline { 2 - 5 } \cline { 4 - 5 } "Ymall & Large & & Small & Large \\
Naming & .86 & .88 & .73 & .59 \\
Mean & .88 & .85 & .56 & .32 \\
\hline
\end{tabular}


only with extralist cues. For targets, the probabilities of correct "yes" and naming responses were both .87 ; for extralist cues these values were .66 and .44 . Naming and "yes/no" performance were identical in the presence of targets, but "yes" judgments showed a strong relative advantage when extralist cues were presented.

This relative advantage of "yes" judgments did not result from a higher level of false recognitions than occurred for naming. For the "yes/no"' response, a false recognition was defined as saying "yes" to a distractor; for the naming response, it was defined as naming a distractor. Probability of a false recognition was relatively higher for extralist cues $(p=.30)$ than it was for targets $(p=.07)[F(1,60)=58.22, M S e=.016]$, but type of response had no effect on the rate of false recognition and did not interact with type of cue (both $F \mathrm{~s}<1$ ). The false recognition rate associated with extralist cues was relatively high, but postexperimental inquiry indicated that this rate was related to the use of category names not intended by the experimenter. For example, if SILK was categorized as "Clothes" instead of "Type of Cloth," the distractor BLOUSE would tend to be falsely recognized.

\section{Discussion}

The results of Experiment 1 are consistent with earlier findings on the effects of natural category size and with the retrieval inhibition explanation for those findings. Set size effects are found when the test stimulus consists of an extralist cue, regardless of the type of response required. That is, when subjects are shown a word from the same category as the target, the size of the category affects memory performance regardless of whether the subjects are making a "yes/no" category decision or whether they are attempting to name the presented item. Set size effects are not found when the test stimulus consists of the target, again regardless of the type of response required. These results indicate that type of cue is critical for obtaining the set size result and that type of response is not. Nevertheless, this is not to say that type of response is unimportant.

The "yes/no" response was associated with higher levels of performance than was naming when extralist cues were presented. This result could be attributed to guessing, since the "yes/no" response requires a binary decision and naming requires specific item recall. However, although the false recognition rate was higher for extralist cues, it did not vary with type of response. Subjects were no more likely to make a false recognition when the "yes/no" response was required than when naming the specific target was required. This comparability suggests that guessing did not play a major role in producing response differences. The "yes/no" superiority is more likely to be based on differences in information. The fact that category size effects were found for both types of responses indicates that the extralist cues were being used to search the category for the presented target. However, with the "yes/no" response, a correct decision could be made by retrieving the target or, if this failed, by remembering that the category defined by the test cue had been studied recently. In contrast, with naming, a correct response could be made only by recovering the studied target.

\section{EXPERIMENT 2}

One purpose of Experiment 2 was to replicate these findings and to explore the effects of presenting the category names during the study trial. Half of the subjects studied each target in the absence of specific category information (as in Experiment 1), and half studied each target juxtaposed with its category name (e.g., Type of Cloth Silk). As before, type of response was manipulated between subjects, and category size was varied within subjects. The presence of the category name was expected to reduce the level of false recognitions, since category uncertainty would be eliminated during study.

The second purpose of Experiment 2 was to determine whether a natural category size effect would be apparent in the presence of a target cue when type of cue was varied within subjects rather than between subjects as in Experiment 1 . With this manipulation, each subject would be confronted with both extralist and target cues, and this procedural change might or might not alter the pattern of the set size effect. If the pattern was unchanged, the ascendancy of type of cue would be supported strongly. Alternatively, having to switch randomly among cue types might occasionally induce subjects to treat targets as extralist cues. If this is the case, category size effects should be apparent even when the subject was confronted with the standard recognition task of responding "yes/no" to a presented target. Hence, category size effects previously found with only extralist cues might also be apparent in recognition under these conditions. Such a result would suggest that the natural category size effect is not uniquely determined by differences between cued recall and recognition or to differences linked to extralist cues and targets as cues. The critical factor may simply be that the testing conditions must encourage search of the defined category so that the concomitant effects of retrieval inhibition will be apparent. Such effects are likely to be more apparent for large than for small categories (e.g., Nelson et al., 1982).

\section{Method}

Design. The design formed a $2^{4}$ mixed-model factorial with presence of the category name and type of response manipulated between subjects and type of test cue and category size varied within subjects.

Materials and Procedure. The materials were identical to those used in Experiment 1. However, the list was subdivided within each condition of category size so that, across subjects, a given target appeared equally often as a target and as an extralist cue. In addition, primacy and recency buffers were omitted. The procedures were the same except that test trial instructions were modified to indicate that some of the test cues would be identical to studied words, some would be meaningfully related to studied words, and some would be completely new. Subjects giving the "yes/no" 
response were told to indicate whether the test word was a studied word or whether it reminded them of a studied word by saying "yes" or "no." Subjects in the naming condition were told that, if the test word reminded them of a studied word, they were to name it, and if not, to say that it was new. Examples were provided for each response type. Finally, the study trial instructions were appropriately modified for subjects receiving category names. They were told to focus on remembering the target in relation to its category name.

Subjects. Sixty-four subjects were assigned to the experiment, with 16 in each between-subjects condition.

\section{Results}

The results are shown in Table 3. These results indicate a pattern that is generally similar to that for Experiment 1 . Fewer items were named (.72) than were correctly judged "yes" (.78), extralist cues (.57) were less effective than target cues (.93), and performance was lower when category size was large (.68) than when it was small (.82). In addition, performance was lower when category cues were absent (.71) than when they were present $(.80)$. Type of response $[F(1,60)=4.97, M S \mathrm{e}$ $=.050]$, type of cue $[F(1,60)=205.67, M S e=.041]$, category size $[F(1,60)=52.98, M S e=.025]$, and cue presence $[F(1,60)=10.56, M S \mathrm{e}=.050]$ were all significant sources.

As before, these effects were qualified by the significance of interactions of type of cue $\times$ category size $[F(1,60)=31.84, M S e=.021]$ and of type of cue $X$ type of response $[F(1,60)=23.18, M S e=.041]$. The interaction with category size is shown in the bottom row of Table 3. As in Experiment 1, deficits in performance were particularly apparent when extralist cues were presented at test and category size was large rather than small. However, in contrast to Experiment 1, a category size effect was also apparent when targets were presented as test cues. Although small in relation to the effect obtained with extralist cues, a Fisher's $L S D$ of .05 indicated that the relatively poorer performance associated with large categories was significant. The reliability of this difference was confirmed in a separate $2^{3}$ analysis of only the target cues, which showed that the only significant source of variance was category size $[F(1,60)=5.22, M S \mathrm{e}=.011]$. Hence, although quite small, the category size effect was apparent when targets were used as cues, even when subjects were providing a "yes/no" recognition decision.

Table 3

Probability Correct as a Function of Type of Test Cue, Category Name Presence During Study, and Type of Response

\begin{tabular}{ccccccc}
\hline & & \multicolumn{3}{c}{ Type of Test Cue } \\
\cline { 3 - 6 } Category & & \multicolumn{2}{c}{ Target } & & \multicolumn{2}{c}{ Extralist Cue } \\
\cline { 3 - 6 } \cline { 5 - 6 } Name & Type of Response & Small & Large & & Small & Large \\
\hline Absent & "Yes/No" & .96 & .92 & & .65 & .38 \\
& Naming & .97 & .93 & & .56 & .24 \\
Present & "Yes/No" & .97 & .88 & .83 & .63 \\
& Naming & .90 & .89 & .70 & .52 \\
& Mean & .95 & .90 & .69 & .44 \\
\hline
\end{tabular}

The interaction between type of cue and type of response simply replicated the pattern found in Experiment 1 in that response differences were found only with extralist cues. For targets, the probabilities of correct "yes" and naming responses were, respectively, .94 and .93 ; for extralist cues, these values were .62 and .51 . As before, correct "yes" judgments showed a strong relative advantage over naming the specific target when extralist cues were presented.

Finally, the only other reliable interaction in the overall analysis was between category name presence at study and type of response $[F(1,60)=4.20, M S e=.041, L S D$ $=.08]$. This interaction indicated that the presence of the category name during study facilitated the "yes/no" response somewhat more than it facilitated the naming response. For the "yes/no" response, the probabilities of correct recognition were .83 and .73 when the category names were, respectively, present and absent; for naming, these values were .75 and .68 .

The level of false recognition in this experiment was somewhat lower overall than in the previous one, and it is difficult to understand how the pattern could be used to explain away the above effects. The statistical analysis indicated that category name presence $(F=1.19)$ and type of response $(F<1)$ had no effect, but that the interaction of these sources was reliable $[F(1,60)=4.59, M S e$ $=0.13, L S D=.08$ ]. For the "yes/no" judgment, the probabilities of a false recognition when the category names were present and absent were, respectively, .10 and .19; for the naming response, they were .14 and .11. Hence, false recognitions were lower for "yes/no" judgments when the category names were present. Adjusting the category name presence $\times$ type of response interaction for false recognitions (hits - false alarms) makes it even more apparent. For the "yes/no" judgment, the adjusted scores for the present and absent conditions were, respectively, .73 and .57 ; for the naming response, they were .61 and .57 . Hence, the presence of the category name during study particularly facilitated "yes/no" decisions. The "yes/no" judgment can be made correctly even when the studied target was not recovered; this would be especially true when the category name was part of the study experience. This finding is consistent with the earlier interpretation offered for the interaction between type of cue and type of response, which is apparent in both experiments. In the "yes/no"'task, a correct decision can be made by either remembering the target or remembering the category, and, in the naming task, a correct response can be made only by retrieving the studied target.

\section{Discussion}

The results of Experiment 2 replicated those of Experiment 1 in finding substantial type of response and category size effects with extralist cues. Furthermore, these effects were uninfluenced by presenting the category name during the study trial. However, unlike Experiment 1, Ex- 
periment 2 also found, regardless of the response required, category size effects with targets as cues. These effects were quite small but the magnitude of retrieval inhibition effects in recognition testing is also quite small (Todres \& Watkins, 1981). Independently of the size of the effect, simply having found it is important for understanding when and why category size makes a difference. It indicates that category size effects are not uniquely determined by the nature of the test task or by the nature of the test cue. The key element seems to be that the conditions of testing must encourage or require search through the category defined by the test cue and that this search is likely to be accompanied by retrieval inhibition linked to the recovery of nontarget instances. In Experiment 2, category search was presumably initiated from target cues because of the presence of extralist cues in the same testing series. Random switching between targets and extralist cues occasionally caused subjects to treat targets as extralist cues. That this can happen indicates that the cue itself is not ascendant over all other considerations. Hence, simply because category search is generally required in the cued-recall task and is only occasionally used in the recognition task, category size effects are more likely to be observed in cued recall with extralist cues than in recognition with targets as cues.

\section{EXPERIMENT 3}

The purpose of Experiment 3 was to replicate the category size effect found with extralist cues in a procedure that involved a recognition discrimination task. Subjects studied targets in the presence of their category names, and, at test, pairs of words were shown. One of the items in each pair was from a studied category, and the other was from an unstudied category (e.g., if WILD ANIMAL TIGER was studied, the test pair could consist of LION TORNADO). Without our mentioning category information, subjects were simply asked to choose the word that seemed most familiar in terms of their study experience.

On the basis of previous findings (e.g., Anisfeld \& Knapp, 1968; Underwood, 1965), recognition was expected to be above chance levels of $50 \%$. The question was whether category size effects would be apparent. Subjects may render the judgment by noting the categories associated with each instance and determining which category appears to be most familiar (e.g., for LION TORNADO, the categories could be WILD ANIMAL and TYPE OF STORM). On the reasonable assumption that the category names were equally accessible for the instances from small and large categories, this process would facilitate recognition for the recently experienced category, but category size effects should not be apparent. Alternatively, subjects may render the judgment by using each instance to search its implied category for the encoded target. In this case, category size effects should be apparent, and the presence of such effects would provide an additional demonstration that these effects are as- sociated with search processes, and not with differences between cued-recall and recognition tasks per se.

\section{Method}

Design. Category size was the only manipulated variable; as in the previous experiments, it was varied within subjects.

Materials and Procedure. The lists and study trial instructions and procedures used in Experiment 2 were also used here. However, at test, subjects were given words from studied and unstudied categories and were told that both words in the pair were new in the sense that the words had not been presented in the study list. They were told that one of them was related to the study experience and that their task was to indicate which word seemed most familiar. When unsure, they were required to guess; after each choice, they called out a number from 1-5, with 1 indicating a "pure guess" and 5 indicating that they were "absolutely sure." The scale was printed on a card and kept in front of the subject during the experiment.

The distractors used in Experiment 2 were also used here, except that they were randomly paired with words from studied categories. Two pairings were created within each list, and both pairings and lists were balanced over subjects. Test stides presented one word above the other such that half of the time the correct item was above and half of the time it was below the distractor.

Subjects. Twenty subjects participated; 10 were assigned to each list, and 5 were assigned to each pairing.

\section{Results and Discussion}

The probabilities of correct identification were, respectively, .87 and .78 for small and large sets. This difference was reliable $[F(1,19)=6.24, M S e=.015]$. In addition, the confidence ratings also showed a significant set size effect $[F(1,19)=46.31, M S e=1,229.24]$. Mean confidence ratings for correct identifications were 4.36 and 3.61 for items belonging to small and large categories. This result indicates that set size effects are obtained when subjects are asked to produce a familiarity discrimination and the test stimulus is an extralist cue. Once again, the critical factor that determines whether or not natural category size effects are apparent is not the task, or the response, but the degree to which the conditions require or encourage search through the related concept set during test.

\section{GENERAL DISCUSSION}

The present findings contribute to an understanding of natural category size effects and thereby contribute to a better understanding of how and when information represented in long-term or semantic memory influences the recall and recognition of episodic information. The presentation of familiar but unrelated words in a memory experiment produces an episodic experience in that the words have some probability of being connected to the general experimental context and with each other. Each presentation, however, is not an isolated event in which only the mental representation corresponding to the presented word is encoded. The presentation of a familiar word activates or primes its representation and other closely related representations existing in its immediate prior knowledge network. For example, the presentation 
of WILD ANIMAL TIGER may activate representations for TIGER, LION, BEAR, etc., and like the representation for TIGER, the related concepts have some probability of becoming encoded and therefore of becoming linked to the episodic experience. The encoding of these related concepts during the study trial reduces the chances of recovering the episodic representation of the target in direct proportion to the number of such concepts. That is, normatively defined natural category size can affect memory for episodic information.

The effect of natural category size, however, is not equally apparent in all retention tests. Both past studies involving between-experiment comparisons and the present studies involving within-experiment comparisons indicate that these effects are more likely to be evident in cued-recall than in recognition tests. Hence, past and present results are inconsistent with the hypothesis that category size effects are produced by encoding weaknesses induced during study (e.g., Nelson, Bajo, \& Casanueva, 1985). This hypothesis assumes that activation spreads from the target representation to related concepts and that the amount of activation any representation receives, including that for the target, is proportional to the number of concepts in the set (see Anderson, 1983). Words having larger sets would receive less activation and would thereby be more weakly encoded in the episodic context, than would words with smaller sets of related concepts. This hypothesis erroneously predicts that natural category size effects should be readily obtained when the test cue consists of either the target itself or an extralist cue, since, no matter what the cue, the target has been more weakly represented during the study experience. Of course, this is not the case, and, at least in its simplest form, the encoding weakness hypothesis can be rejected as an explanation for target set size effects.

Past and present results are more consistent with the retrieval inhibition explanation. This explanation assumes that natural category size effects are due to inhibitory processes that operate during search, and therefore any cue, task, or set of testing conditions that requires or encourages searching the category should show these effects. The results of Experiments 1-3 indicate that category size effects were evident whenever extralist cues were presented, regardless of the nature of both the required response and task. Hence, memory for instances from larger categories was differentially poor in the standard cued-recall task requiring naming (Experiments 1 and 2), in the category recognition task requiring "yes/no" judgments of category reoccurrence (Experiments 1 and 2), and in the recognition discrimination task requiring judgments of familiarity (Experiment 3 ). When extralist cues are presented at test, natural category size effects transcend differences in task and response requirements. With these cues, the target itself is absent and subjects are required to search memory for information that will lead to it. This search is likely to be associated with the inhibitory effects of retrieval inhibition that occur when nontarget items are recovered, and these inhibitory effects should be greater when there are more items in the set (Nelson et al., 1982; Raaijmakers \& Shiffrin, 1981; Watkins, 1979).

The retrieval inhibition principle is reaffirmed in the contrasting results of Experiments 1 and 2 involving targets as cues. In Experiment 1, type of cue was varied between subjects, and, as in many previous recognition experiments (Nelson, Canas, Casaneuva, \& Castano, 1985), category size had no effect whatever. The recognition response presumably was mediated by familiarity and by retrieving episodic links to context and to other list items (Gillund \& Shiffrin, 1984; Mandler, 1980). In Experiment 2 , however, type of cue was varied within subjects, and category size had a small reliable effect. Presumably, recognition was primarily mediated by the same processes in both experiments, but when each subject experienced both targets and extralist cues in a random sequence, targets must have occasionally been treated as extralist cues. Such treatment would be most likely when the target was not immediately recognized, and on such occasions, it would be used to initiate search of the category. With this search, the effects of category size and the concomitant effects of retrieval inhibition were evident.

The hypothesis that natural category size effects are contingent on a cue-induced search process should not be taken to mean we think that search is not involved in recognition because category size effects are not usually found in this task. There is ample evidence indicating that search can be an important process in recognition (e.g., see Gillund \& Shiffrin, 1984; Mandler, 1980). However, when search is involved in cued recall and recognition, subjects are presumably searching for different kinds of information. When extralist cues are presented at test, subjects engage retrieval operations that are primarily directed toward related concepts in long-term memory that are defined by the test cue. Searching through concepts within these sets provides information leading to target recovery (Nelson et al., 1982). When search occurs in recognition, it seems to involve a search for contexts associated with the encoding experience (e.g., Mandler, 1980). Hence, search processes are presumably involved in both tasks with interconnections to prior knowledge, and to episodically encoded information contributing differentially to performance.

Finally, note that finding category size effects in a recognition task is important, since it indicates that the critical factor is not the type of cue itself. What is critical is not whether the task involves target or extralist cues, whether the task involves a "yes/no" or naming response, or whether the task requires cued recall or recognition; it is that the testing conditions must require or encourage search through the category of information specified by the test cue. When such a search is initiated, information represented in long-term or semantic memory is likely to influence memory for episodic information. Furthermore, under these conditions, this influence is likely to be negative. As in experiments on cue overload (Watkins, 1979) and on the FAN effect (Anderson, 1983), the more 
potential targets there are, the worse the chances are for recovering a recently experienced target experience. The primary difference between cue overload and FAN experiments and the present experiments is in whether the source of the interference arises from what is directly learned in the experimental context or from knowledge acquired prior to the laboratory experience.

\section{REFERENCES}

ANDERson, J. R. (1983). A spreading activation theory of memory. Journal of Verbal Learning \& Verbal Behavior, 22, 261-295.

ANDERson, J. R., \& BowER, G. H. (1974). A propositional theory of memory. Memory \& Cognition, 2, 406-412.

ANISFELD, M., \& KNAPP, M. E. (1968). Association, synonymity, and directionality in false recognition. Journal of Experimental Psychology, 77, 171-179.

COFER, C. N. (1958). Comparison of word associations obtained by the methods of discrete single word and continued association. Psychological Reports, 4, 507-510.

Collins, A. M., \& LofTus, E. F. (1975). A spreading-activation theory of semantic processing. Psychological Review, 82, 407-428.

Gillund, G., \& SHIFFrin, R. M. (1984). A retrieval model for both recognition and recall. Psychological Review, 91, 1-67.

Joelson, J. M., \& Herrman, D. J. (1978). Properties of categories in semantic memory. American Journal of Psychology, 91, 101-114.

MANDler, G. (1980). Recognizing: The judgment of previous occurrence. Psychological Review, 87, 252-271.

McEvoy, C. L., NELSON, D. L. (1982). Category and instance norms for 106 categories of various sizes. American Journal of Psychology, 95, 581-634.

Meyer, D. E., \& SchVANeveldt, R. W. (1971). Correlated operation in searching stored semantic categories. Journal of Experimental Psychology, 90, 227-234.

Nelson, D. L. (1981). Many are called but few are chosen: The influence of context on the effects of category size. In G. H. Bower
(Ed.), The psychology of learning and motivation (Vol. 15, pp. 129162). New York: Academic Press.

Nelson, D. L., \& Bajo, M. T. (1985). Prior knowledge and cued recall: Category size and dominance. American Journal of Psychology, 98 , 503-517.

Nelson, D. L., Bajo, M. T., Casanueva, D. M. (1985). Prior knowledge and memory: The influence of natural category size as a function of intention and distraction. Journal of Experimental Psychology: Learning, Memory, \& Cognition, 11, 94-105.

Nelson, D. L., Canas, J., Casanueva, D. M., \& Castano, D. (1985). Prior knowledge and recognition. American Journal of Psychology, 98, 379-397.

Nelson, D. L., \& McEvoy, C. L., \& Friedrich, M. A. (1982). Extralist cuing and retrieval inhibition. Joumal of Experimental Psychology: Learning, Memory, \& Cognition, 8, 89-105.

RaAiJMakers, J. G. W., \& Shiffrin, R. M. (1981). Search of associative memory. Psychological Review, 88, 93-134.

Roediger, H. L. (1974). Inhibiting effects of recall. Memory \& Cognition, 2, 261-269.

Smith, S. M., Glenberg, A. M., \& Bjork, R. A. (1978). Environmental context and human memory. Memory \& Cognition, 6, 342-353.

THORNDIKE, E. L., \& LORGE, I. (1944). A teacher's word book of 30,000 words. New York: Teachers College Press, Columbia University.

Todres, A. K., \& WaTKINs, M. J. (1981). A part-set cuing effect in recognition memory. Joumal of Experimental Psychology: Human Learning and Memory, 7, 91-100.

Tulving, E. (1983). Elements of episodic memory. New York: Oxford University Press.

UNDERWOOD, B. J. (1965). False recognition produced by implicit verbal responses. Journal of Experimental Psychology, 70, 122-129.

WatkINs, M. J. (1979). Engrams as cuegrams and forgetting as cue overload: A cueing approach to the structure of memory. In C. R. Puff (Ed.), Memory organization and structure. New York: Academic Press.

(Manuscript received April 29, 1986; revision accepted for publication August 19, 1986.) 